\title{
Correction to: Automated Machine Learning for Satellite Data: Integrating Remote Sensing Pre-trained Models into AutoML Systems
}

Nelly Rosaura Palacios Salinas, Mitra Baratchi (D), Jan N. van Rijn (D), and Andreas Vollrath

Correction to:

Chapter "Automated Machine Learning for Satellite Data: Integrating Remote Sensing Pre-trained Models into AutoML Systems" in: Y. Dong et al. (Eds.):

Machine Learning and Knowledge Discovery in Databases, LNAI 12979, https://doi.org/10.1007/978-3-030-86517-7_28

The given name and surname of the author Nelly Rosaura Palacios Salinas have been wrongly attributed in some parts of the original publication. This has now been corrected. 